Chen Lunjin (Orcid ID: 0000-0003-2489-3571)

Liu Xu (Orcid ID: 0000-0002-7211-0546)

Tong Yuguang (Orcid ID: 0000-0002-3354-486X)

Pu Zuyin (Orcid ID: 0000-0002-8458-6648)

Zong Qiu-Gang (Orcid ID: 0000-0002-6414-3794)

Sun Weijie (Orcid ID: 0000-0001-5260-658X)

\title{
Modulation of Whistler Mode Waves by Ion Scale Waves Observed in the Distant Magnetotail
}

\author{
Duo Zhao ${ }^{1}$, Suiyan $\mathrm{Fu}^{2}$, George K. Parks ${ }^{3}$, Lunjin $\mathrm{Chen}^{4}$, Xu Liu ${ }^{4}$, Yuguang \\ Tong ${ }^{3}$, Jinxing $\mathrm{Li}^{5}$, Zuyin $\mathrm{Pu}^{2}$, Qiugang Zong', Weijie Sun ${ }^{6}$, Shouxian $\mathrm{Li}^{1}$, and \\ JianZhu An ${ }^{1}$
}

\author{
${ }^{1}$ Institute of Applied Physics and Computational Mathematics, Beijing, China. \\ ${ }^{2}$ School of Earth and Space Sciences, Peking University, Beijing, China. \\ ${ }^{3}$ Space Sciences Laboratory, University of California, Berkeley, California, USA. \\ ${ }^{4}$ William B. Hanson Center for Space Sciences, University of Texas at Dallas, \\ Richardson, Texas, USA. \\ ${ }^{5}$ Department of Atmospheric and Oceanic Sciences, University of California, Los \\ Angeles, CA, USA. \\ ${ }^{6}$ Department of Climate and Space Sciences and Engineering, University of Michigan, \\ Ann Arbor, Michigan, USA.
}

Corresponding author: Suiyan Fu (Suiyanfu@pku.edu.cn)

This is the author manuscript accepted for publication and has undergone full peer review but has not been through the copyediting, typesetting, pagination and proofreading process, which may lead to differences between this version and the Version of Record. Please cite this article as doi: 10.1029/2019JA027278

This article is protected by copyright. All rights reserved. 


\section{Key Points:}

- Modulation of whistler mode waves by ion scale waves are observed in the distant magnetotail.

- The whistler mode waves are excited by field-aligned electron populations generated by the ion scale waves.

- The ion scale waves, possibly kinetic Alfven waves, are driven by tailward streaming ion beams. 


\begin{abstract}
Wave activities in tailward flows have been explored in the distant magnetotail at $\sim 54 R_{\mathrm{E}}$, where the coupling between whistler mode waves and ion scale waves was observed. The whistler mode waves periodically appeared at each cycle of the ion scale waves, and the electron distribution functions associated with the whistler mode waves showed enhancement in the direction parallel to background magnetic field. Wave analyses show that the field-aligned electron components act as the energy source of the whistler mode waves. The ion scale waves are generated by the interaction of the hot ion beam with background ions. A likely candidate of the ion scale wave is the kinetic Alfven wave, which can generate the enhanced field-aligned electron populations by the parallel electric field.
\end{abstract}

\title{
Plain Language Summary
}

The ionized particles constrained by the earth’s magnetic field involve dynamics of different spatial and temporal scales. Because of large mass difference, the scales characterizing the ion and electron dynamics are very different, and the related processes are often considered separately. In this study, we have found the electron scale waves can be modulated by ion scale waves, indicating that the dynamics of electrons and ions are coupled. The ion scale waves are induced by the streaming ion beams superposed on the background ions. In the process, electrons are accelerated parallel to the magnetic field direction by the ion scale wave, resulting in the deviation of electron populations from the equilibrium state. The unstable electrons then release the excess energy by generating electron scale waves, called whistler mode waves. 


\section{Introduction}

The plasma sheet [Bame et al., 1967] in the Earth's magnetotail is a region full of plasma activities characterized by high-speed plasma flows [Baumjohann et al., 1990; Angelopoulos et al., 1992], magnetic structures [Nakamura et al., 2002; Slavin et al., 2003], wave activities [Khotyaintsev et al., 2011; Zhao et al., 2016], and particle accelerations [Zhou et al., 2010; Fu et al., 2011]. During the active times in the plasma sheet, both earthward and tailward flows are generated [Nagai et al. 1998]. The earthward flows carry energy and magnetic flux to the inner magnetosphere and produce various space physics phenomenon, like energetic particle injection [Sergeev et al., 2012; Gabrielse et al., 2017], geomagnetic pulsations [Runov et al., 2014], and brightening of auroras [Donovan et al. 2008]. The tailward flows stream down the magnetotail to distances of $\sim 60 R_{\mathrm{E}}$ and beyond [Artemyev et al., 2017; Kiehas et al., 2018], where the wave and particle behaviors show unique signatures for different plasma environment and magnetic field configuration [Li et al., 2014; Zhou et al., 2015; Zhao et al., 2017]. However, the plasma dynamics in the distant magnetotail have been seldomly explored compared to the near earth regions.

During the plasma sheet activities, whistler mode waves are often observed in bursty bulk flows where the magnetic flux is compressed [Viberg et al., 2014; Zhang et al., 2018]. Actually, the whistler mode wave exists throughout the magnetosphere [Wilson et al., 2011; Li et al., 2013]. In the inner magnetosphere, the whistler mode waves often appear as broadband hiss [Summers et al., 2014] and chorus waves with rising and falling tone structures [Sazhin and Hayakawa, 1992; Keika et al., 2012]. The pitch angle scattering of the electrons by the whistler mode waves could precipitate the radiation belt electrons into the ionosphere [Thorne et al., 2010; Mozer et al., 2017; Li et al., 2019], and results in the loss of electrons from the plasma sheet and carry energy away [Li et al., 2013; Zhang et al., 2019a]. 
Besides interaction with electrons, whistler mode waves could also be modulated by low frequency waves since the inhomogeneity of the plasma density and magnetic field could influence the generation and propagation of this wave [Karpman and Kaufman, 1982; Streltsov et al., 2006]. In the radiation belt region, whistler mode waves can be modulated by compressional ULF waves, which induce electron density, magnetic field and resonant electron variations [ $\mathrm{Li}$ et al., 2011; Zhang et al., 2019b]. Tenerani et al. [2012] have shown that ion scale solitary waves can trap the whistler mode waves at the wave valley or hump, and this process can connect the electron and ion scale dynamics. In addition, Colpitts et al., [2016] have reported observations of coupling between whistler mode wave and electromagnetic ion cyclotron waves. However, the modulation mechanisms of whistler mode waves we present in this paper have not been addressed previously.

The whistler mode waves have been extensively explored in the near earth region [Viberg et al., 2014; Tyler et al., 2019], but not in the distant magnetotail where the plasma environment is very different. This paper presents observations of whistler mode waves at $\sim 60 R_{\mathrm{E}}$ down the geomagnetic tail. These waves are periodically excited by electron distribution functions modulated by low frequency waves on the ion dynamic scale. To the best of our knowledge, the characteristic signatures we will show have not been reported in the magnetotail region, and they are important for enhancing our understanding of cross-scale wave interaction mechanisms in plasmas.

\section{Observations}

The ARTEMIS (Acceleration, Reconnection, Turbulence, and Electrodynamics of the Moon's Interaction with the Sun) mission [Angelopoulos, 2011] is an expansion of the THEMIS (Time History of Events and Macroscale Interactions during 
Substorms) mission [Angelopoulos, 2008] with two spacecraft placed at the lunar orbit, making it possible to explore the magnetotail at $\sim 60 R_{\mathrm{E}}$. The data used in this paper come from the Fluxgate Magnetometer (FGM) [Auster et al., 2008], Electrostatic Analyzer (ESA) [McFadden et al., 2008], Solid State Telescope (SST) [Angelopoulos, 2008], and the Search Coil Magnetometer (SCM) [Roux et al., 2008]. Wave spectra are calculated onboard the spacecraft by Fast Fourier Transforms (FFTs) [Cully et al., 2008]. All quantities in this paper are presented in the Geocentric Solar Magnetospheric (GSM) System, unless otherwise noted.

On May 24, 2013, ARTEMIS P1 probe was located at $(-54,8.3,2.3) R_{\mathrm{E}}$ in the distant magnetotail, where a tailward plasma flow and fluctuating magnetic field were detected (Figure 1). The flow, which begins at 032930 UT (Figure 1b), was associated with an anti-dipolarization front [Nakamura et al., 2002; Li et al., 2014] near the leading edge at $\sim 033000 \mathrm{UT}$ (Figure 1a). Inside the plasma flow, the magnetic field shows large amplitude fluctuations (Figure 1a), indicating presence of active plasma dynamics. The ion (Figure 1c) and electron (Figure 1d) spectra in the flow are similar to that in the plasma sheet [Bame et al., 1967]. From 033100UT to $\sim 033200$ UT (blue line), the ion temperature showed a much hotter component parallel to the magnetic field (Figure 1f), and resulted in the temperature anisotropy $T_{\|} / T_{\perp}$ exceeding 1.0 and approaching 2.0 at some point (Figure 1g). During this interval, the ion beta $\beta_{i}$, which is defined as the ratio of ion thermal pressure to magnetic pressure, increased to 10 (Figure 1h). The behavior of wave activities observed during this interval is the major interest of this study. 


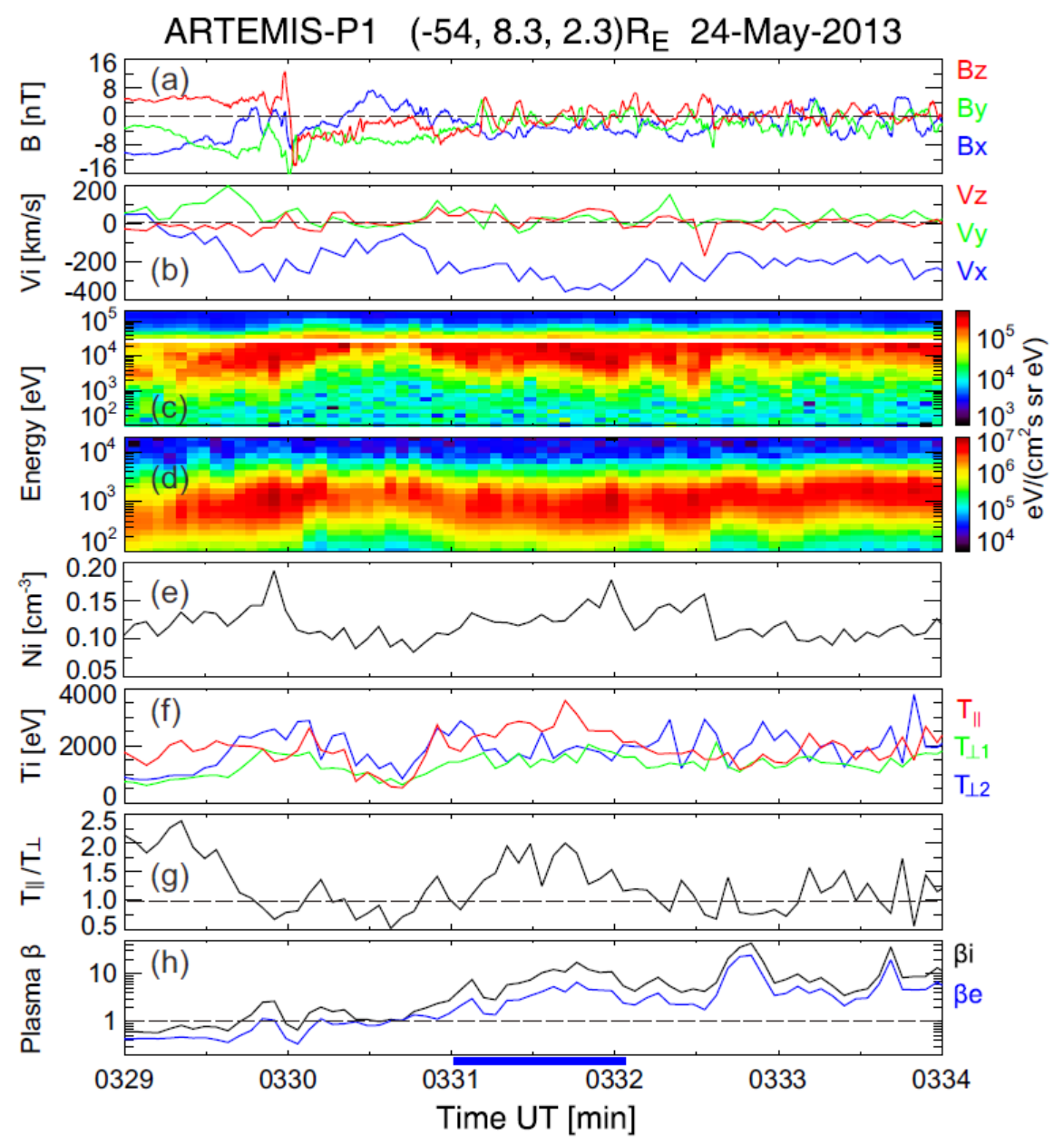

Figure 1. Summary plot of ARTEMIS-P1 observations in the distant magnetotail. (a) Magnetic field, (b) Ion bulk velocity, (c) Ion and (d) electron differential energy flux, (e) Ion density, (f) Ion temperature in the field-aligned coordinate system, (g) Ion temperature anisotropy, (h) Ion beta ( $\beta_{i}$ black line) and electron beta ( $\beta_{e}$ blue line). The blue horizontal line below (h) marks the time interval presented in figure 2. All the data recorded by the ESA instrument are under burst mode.

The wave activities of different frequencies were analyzed in detail from 033103UT to 033205UT as presented in Figure 2. Large amplitude waves of $\sim 6 \mathrm{nT}$ have been observed from the magnetic field fluctuations (Figures 2a and 2b), and each 
cycle of the wave was marked as 1-6 in Figure 2a. The average frequency of this wave is about $0.1 \mathrm{~Hz}$ in the spacecraft frame, close to the local ion cyclotron frequency $f_{c i} \sim 0.075 \mathrm{~Hz}$, hence we call this wave ion scale wave. Figure 2c shows the high-frequency magnetic field fluctuations filtered above $10 \mathrm{~Hz}$ and Figure 2d shows the power spectrum of the waves calculated onboard the spacecraft with the white line indicating 0.4 times the local electron cyclotron frequency $\left(f_{c e}\right)$. A clear signature is that the high frequency waves appeared periodically at each cycle of the ion scale wave (except cycle 4), and we interpret them as modulation by the ion scale waves.

The high frequency waves have been identified as the lower-band whistler mode waves, because they are nearly right-hand circularly polarized (not shown) with a frequency lower than $0.5 f_{c e}$ (figure $2 \mathrm{~d}$ ). These waves propagated with angles from $5^{\circ}$ to $34^{\circ}$ respect to the local magnetic field direction. The electron distribution functions associated with the whistler mode waves showed anisotropies in the direction parallel and antiparallel to the magnetic field. We measured these anisotropies by $\left(F_{+\|}-F_{-\|}\right) / F_{-\|}$as shown in Figure 2e, where $F_{+\|}\left(F_{-\|}\right)$is the particle flux parallel (antiparallel) to the background magnetic field direction. The wave emissions are associated with the parallel particle flux enhancement of the electrons with energy from $\sim 1 \mathrm{keV}$ to $\sim 10 \mathrm{keV}$ (the flux anisotropy of third whistler mode wave is weakened after normalization), while the anisotropies are not as pronounced outside the wave intervals. The transverse flux anisotropy of $F_{\perp} / F_{\|}\left(F_{\perp}\right.$ is the perpendicular particle flux and $F_{\|}$is the average particle flux in parallel and anti-parallel directions) is also calculated in figure $2 \mathrm{f}$, and there are no recognizable anisotropies when the waves appear. Thus, the increased parallel electron fluxes are suggested to be the energy source of the whistler mode waves as discussed in the next section. 
The hodograms of the ion scale waves are shown in the boxes below Figure 2f, and the numbers 1-6 correspond to the six wave cycles. The first and second cycles are circularly and elliptically left-hand polarized in the spacecraft frame while the other cycles are irregularly polarized. An interesting feature to note is that the first and second ion scale wave cycles are associated with whistler mode waves of rising and falling band structures in the wave spectrum (figure 2d), while the whistler mode waves corresponding to other cycles are structureless. Although the magnetic field strength during the first whistler mode wave (033108UT-033112UT) increased from $\sim 6 \mathrm{nT}$ to $\sim 8 \mathrm{nT}$, the wave frequency enhancement from $\sim 30 \mathrm{~Hz}$ to $\sim 80 \mathrm{~Hz}$ is much larger and seems not to be formed by the strengthening of the magnetic field. Since the resolution of the wave data during this interval was not sufficient, and the single probe detection could not distinguish spatial and time variations, the detail mechanisms are still awaiting for further exploration. The wave features of the first two cycles indicate nonlinear coherent processes that connect the electron and ion scale dynamics have taken place. 

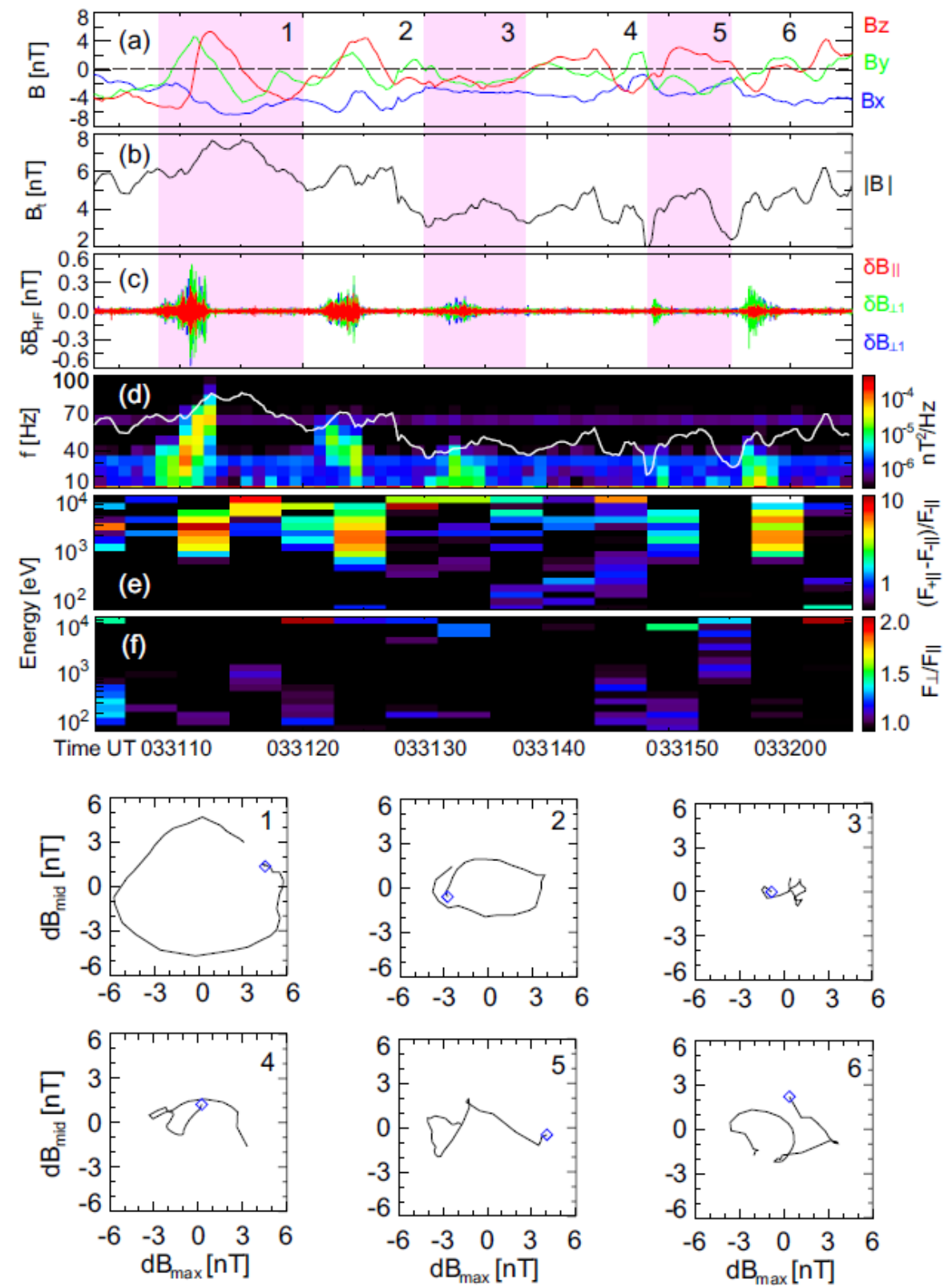

Figure 2. Wave analyses and corresponding particle behaviors. (a) Magnetic field components, (b) Total magnetic field strength, (c) Magnetic field fluctuations filtered above $10 \mathrm{~Hz}$ in field-aligned coordinate system, (d) magnetic field wave spectrum with the white line that marks $0.4 f_{c e}$, (e) Electron flux anisotropy in parallel and antiparallel directions calculated from the parallel electron differential flux $\left(F_{+\|}\right.$, pitch 
angle: $\left.0^{\circ}-30^{\circ}\right)$ minus the antiparallel electron differential flux $\left(F_{-\|}\right.$, pitch angle: $150^{\circ}-180^{\circ}$ ) and then normalized by $F_{-\|}$. (f) electron flux anisotropy between perpendicular $\left(F_{\perp}\right.$, pitch angle: $\left.60^{\circ}-120^{\circ}\right)$ and parallel $\left(F_{\|}=\left(F_{+\|}+F_{-\|}\right) / 2\right)$ directions calculated by $F_{\perp} / F_{\|}$. The pink and white colors separate the six wave cycles marked by 1-6 in panel (a). The boxes marked by 1-6 below panel (f) are the hodograms corresponding to 1-6 of the wave cycles. The normal direction of the hodograms points out of the paper and the blue diamond is the starting point.

\section{Particle distributions and wave analysis}

To study the generation mechanism of the whistler mode waves and ion scale waves, linear theory of hot plasma dispersion analyses based on the particle distribution functions have been employed with PDRK kinetic dispersion relation solver [Xie and Yong, 2016], and the major results are also checked with WHAMP method [Ronnmark, 1982]. The electron and ion distribution functions are fitted by multi-Maxwellian shaped distributions to make computation. When fitting the particle distributions, a cold component will be added if necessary, to keep the fitted density the same as the measured value, and the drift velocity of all the populations have been shifted together to keep zero current in the system. The analysis of the first and fifth whistler mode waves are shown in Figure 3 and Figure 4, while Figure 5 shows the results of the ion scale waves.

The electron distribution function associated with the first whistler mode wave (Figure 3a) exhibits a higher electron phase space density (PSD) in the parallel direction than in the antiparallel direction. To extract the enhanced component, the net population is calculated as the parallel PSD minus the antiparallel value, as shown in 
Figure 3b. The enhanced electrons are diffused on the right side of the phase space (Figure $3 b$ ) and could not be represented by a single beam, hence multi-component drifting bi-Maxwellian distributions have been used to fit this population (Figure 3c). The fitting results in parallel and perpendicular directions are shown in Figure 3d, and the fitting parameters are listed in Table 1 in the first set.

Wave dispersion analysis based on the fitted parameters shows an anti-parallel propagating mode as displayed in Figure 3e. The growth rate (blue line) of this wave mode shows two positive peaks at the frequency (black line) of 0.16 and 0.23 times the electron cyclotron frequency $\left(\omega_{c e}\right)$. To verify the contributions to these two peaks, the four beams of the field-aligned population are removed in turn to calculate the growth rate. As shown in Figure 3f, the absence of beam 1 (black will) will not influence the growth rate, while the disappearance of beam 2 or beam 3 (blue line) will result in the absence of the second peak and shift the wave number of the first peak, indicating the second peak of the growth rate is contributed by beam 2 and beam 3 together. When beam 4 is removed (red line), the first peak disappeared, but the second peak became much larger, and this implies that the first peak of the growth rate is contributed by beam 4 , and beam 4 could also suppress the instabilities induced by beam 2 and beam 3. The temperature anisotropies of the hot electrons (Figure 3d) also support a weak parallel propagating mode, but the growth rate of this mode is 30 times lower than the mode induced by the parallel enhanced populations and could hardly induce waves. 

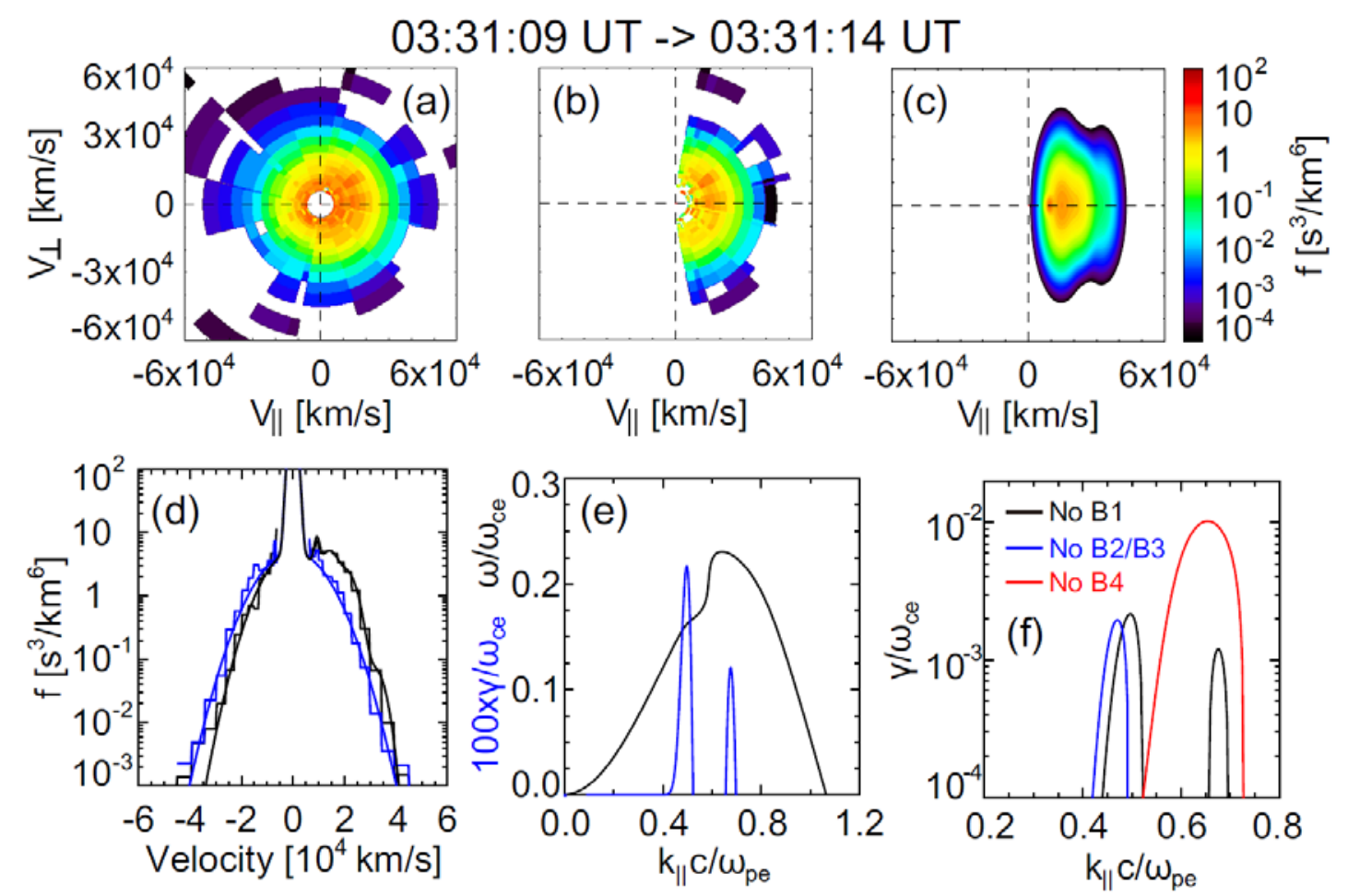

Figure 3. Electron distributions and wave dispersion studies of whistler mode wave 1. (a) 2D electron velocity distribution functions from 033109UT to 033114UT, where $V_{\|}$is the velocity parallel to the magnetic field $(\boldsymbol{B}), V_{\perp}$ is the velocity perpendicular to $\boldsymbol{B}$ and the ion bulk velocity. (b) Parallel enhanced electrons calculated by the parallel electron phase space density minus the antiparallel value, (c) Fitting results of the parallel increased electrons, (d) 1D cut (step lines) and fitting (curve line) result of the electron distributions in parallel (black line) and perpendicular (blue line) directions, (e) Frequency $(\omega)$ and growth rate $(\gamma)$ as a function of parallel wave number $\left(k_{\|}\right)$calculated based on the fitted electron distribution functions, $\omega$ and $\gamma$ are normalized by $\omega_{c e}$ and $k_{\|}$is normalized by electron plasma frequency $\left(\omega_{p e}\right)$ versus the speed of light $(c)$, (f) Growth rate calculated for the electron distribution functions without beam 1 (Black line), beam 2 or beam 3 (Blue line), and beam 4 (Red line). 
The electron distribution functions of the fifth whistler mode wave have also been analyzed with the same method and shown in Figure 4. During this interval, the field-aligned population is also shown (Figure 4b), and the background electrons are isotropic (Figure 4d). The fitting results are given in the second set of Table 1, based on which wave dispersion analyses are employed. In this case, the distribution also supports an anti-parallel propagating mode with a peak growth rate at the frequency of $0.045 \omega_{c e}$ (Figure 4e). To study the influences of the parameters of the field-aligned population to the wave growth, the density and temperature anisotropy of all the beams are changed to explore their roles. As shown in figure 4f, when the density of the field-aligned component increased from 0.5 to 3 times the initial value (shown by solid line with different colors), the growth rate and the corresponding parallel wave number $k_{\|}$enhanced. The temperature anisotropy is changed by adjusting the perpendicular temperature of all beams (green and red dash lines). When the perpendicular temperature is half of the current distribution, no positive growth rate is shown, indicating the excitation of this wave needs to exceed a threshold of beam temperature anisotropy. As expected, a higher temperature anisotropy with hotter perpendicular component will result in the strengthening of the growth rate and possibly the appearance of other growth rate peaks. For other whistler mode waves, the fitted parallel enhanced population of the second and fourth waves are unstable, while the distribution of the third wave did not provide positive growth rate, and this might be due to the limited time resolution of the particle instrument that could not fully record the parallel enhanced components. 

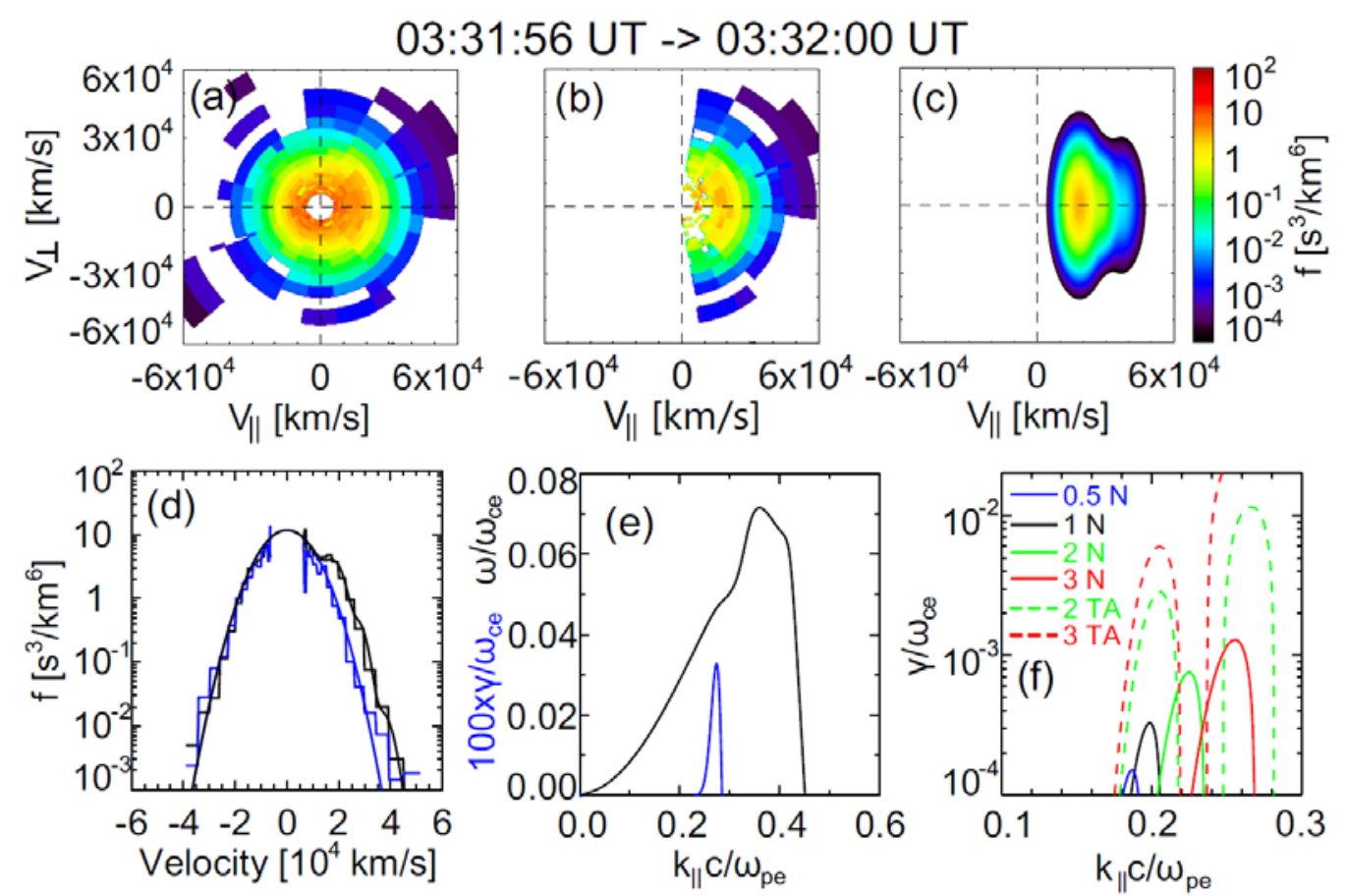

Figure 4. Electron distributions and wave dispersion studies of whistler mode wave 5. (a) - (e) Analysis of the electron distributions from 033156 UT to 033200 UT with the same format as in Figure 3, (f) Growth rate calculated with 0.5 (Blue solid line), 1 (Black solid line), 2 (Green solid line), 3 (Red solid line) times the density and 2 (Green dash line), 3 (Red dash line) times the temperature anisotropy $\left(T_{\perp} / T_{\|}\right)$of the field-aligned populations of the electron distribution functions.

Table 1. Fitting results of the electron and ion distribution functions. The parameters include density $\mathrm{N}$, beam streaming velocity $V_{0}$, parallel temperature $T_{\|}$, and ratios of perpendicular to parallel temperature $T_{\perp} / T_{\|}$.

\begin{tabular}{|c|c|c|c|c|}
\hline \multicolumn{6}{|l|}{033109 UT - 033114 UT, $|\boldsymbol{B}|=6.5 \mathrm{nT}$, Whistler mode wave } \\
\hline Components & $\mathrm{N}\left(\mathrm{cm}^{-3}\right)$ & $V_{0}(\mathrm{~km} / \mathrm{s})$ & $T_{\|}(\mathrm{eV})$ & $T_{\perp} / T_{\|}$ \\
\hline Hot electrons & 0.0435 & -4130.4 & 384 & 1.4 \\
\hline
\end{tabular}




\begin{tabular}{|c|c|c|c|c|}
\hline Cold electron & 0.0258 & -4130.4 & 10 & 1.0 \\
\hline Electron beam 1 & 0.0004 & 5204.6 & 5 & 6.0 \\
\hline Electron beam 2 & 0.0155 & 10258.6 & 50 & 10.0 \\
\hline Electron beam 3 & 0.0068 & 17166.6 & 60 & 6.0 \\
\hline Electron beam 4 & 0.0003 & 28045.6 & 55 & 10.0 \\
\hline Ions & 0.0923 & 0 & 2000 & 1.0 \\
\hline \multicolumn{5}{|c|}{033156 UT -- 033200 UT, $|\boldsymbol{B}|=4.5$ nT, Whistler mode wave } \\
\hline Hot electrons & 0.11 & -2011.3 & 404 & 1.0 \\
\hline Electrons beam 1 & 0.012 & 16532.7 & 59 & 8.0 \\
\hline Electrons beam 2 & 0.00075 & 25817.7 & 41 & 8.0 \\
\hline Electrons beam 3 & 0.00010 & 34862.7 & 64 & 8.0 \\
\hline Ions & 0.12285 & 0 & 2000 & 1.0 \\
\hline \multicolumn{5}{|c|}{ 03:31:00 UT -- 03:32:05 UT, $|\boldsymbol{B}|=4.9$ nT, Ion scale wave } \\
\hline Hot Ion & 0.047 & -189 & 2219 & 1.56 \\
\hline Cold Ion & 0.059 & -189 & 100 & 1.0 \\
\hline Ion Beam & 0.026 & 780 & 2346 & 1.66 \\
\hline Electrons & 0.132 & 0 & 487 & 1.0 \\
\hline
\end{tabular}

The field-aligned enhanced electron populations appear periodically with the ion scale waves (Figure 2e), hence we suggest they are most likely generated by the ion scale waves. The excitation of the ion scale wave has also been studied with particle data. The 2D ion velocity distributions and $1 \mathrm{D}$ cuts along the parallel direction in the plasma flow frame during the time of Figure 2 are shown in Figures $5 \mathrm{a}$ and 5b. The ion velocity distribution function shows that the phase space densities of the parallel streaming ions are much larger than that in the opposite direction. Maxwell fitting analyses show that the ion distribution consisted of a hot population (black dash line 
in Figure 5b) and a field-aligned ion beam (blue dash line) streaming with a velocity of $\sim 960 \mathrm{~km} / \mathrm{s}$, which is about 3.2 times the local Alfven velocity $\left(V_{A}=297 \mathrm{~km} / \mathrm{s}\right)$. The fitting parameters are listed in Table 1 of last sets.

Wave analyses have been performed based on the fitting parameters, and two modes were found to be unstable. The first mode is excited by the ion beam, and have a maximum growth rate when the wave vector is parallel to the magnetic field as shown in Figure 5c. The growth rate of this mode has a wide range of positive values covering the frequency from very low to $\sim 2 \omega_{c i}$, and shows a peak at the frequency of $\sim 0.65 \omega_{c i}$. The black dash line marks the ion Alfven speed in the $k-\omega$ plane, and the phase velocity of this wave is close to $V_{A}$ if the frequency is around $\omega_{c i}$. The second mode shown in Figure $5 d$ is a low frequency $\left(\omega<0.1 \omega_{c i}\right)$ wave that propagated quasi-parallel to the magnetic field and generated by the temperature anisotropy of the hot ion population. Since the drift velocity of the hot ion population is low $\left(0.16 V_{A}\right.$ according to fitting result, as shown Figure $\left.5 \mathrm{~b}\right)$, and the flow velocity is mostly contributed by the ion beam, the Doppler shift effect of the ion scale waves should not be large. Considering that the frequency of the ion scale wave is close to $\omega_{c i}$, it is more likely to be induced by the first mode with the higher frequency generated by the ion beams. In observations, the fluctuations of the ion scale waves were mainly in the perpendicular direction (Figure 2a), and this resulted in a quasi-parallel propagation mode that is consistent with the wave analyses. 

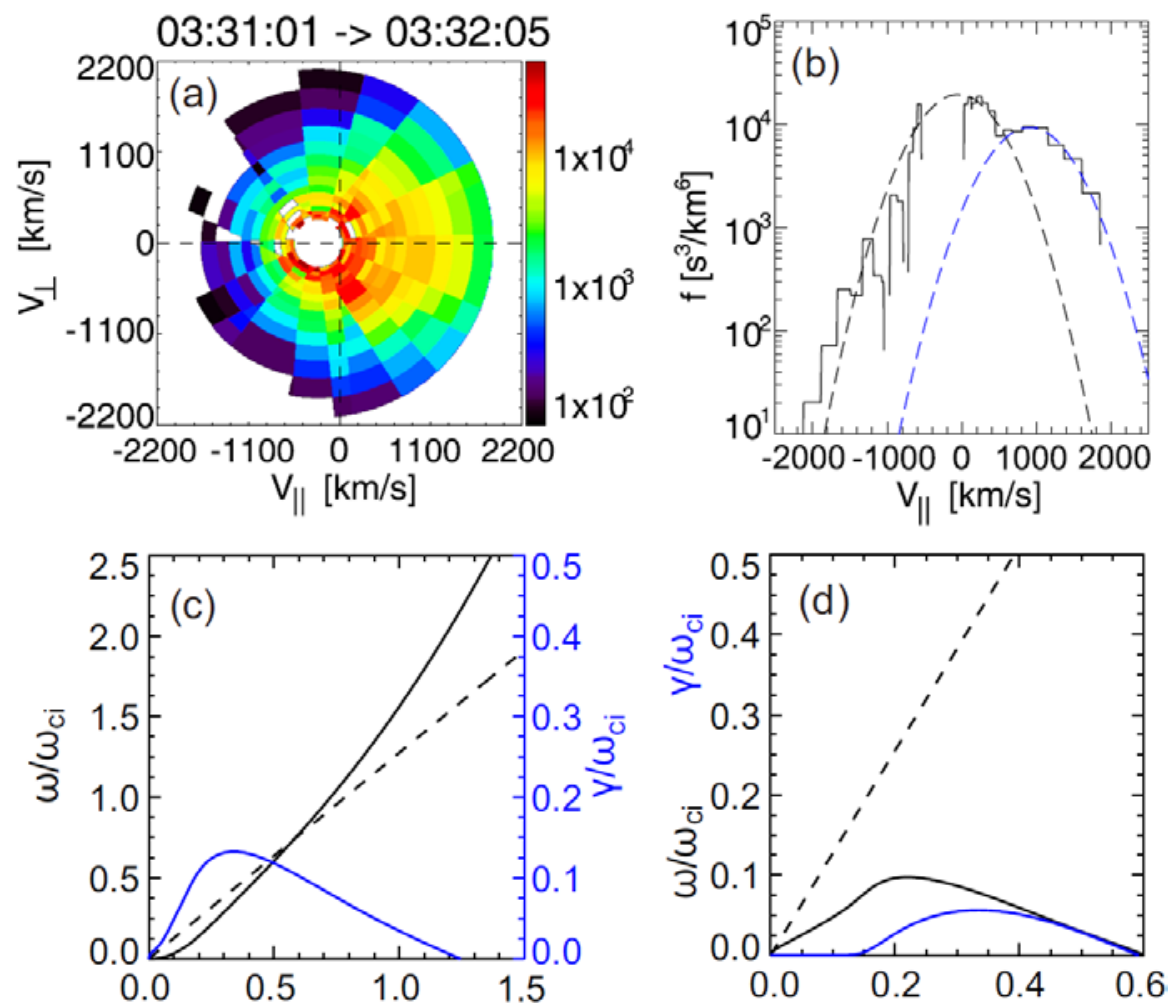

Figure 5. Ion distribution and wave dispersion studies of the ion scale wave. (a) Ion 2D velocity distribution function from 03:31:00 UT to 03:32:05 UT in the plasma flow frame. $V_{\|}$is the velocity parallel to the magnetic field $(\boldsymbol{B}), V_{\perp}$ is the velocity perpendicular to $\boldsymbol{B}$ and the ion bulk velocity, (b) 1D cuts of ion distribution functions along $\boldsymbol{B}$ (black line). The black dash line shows Maxwell fitting of the background ions, while the blue dash line marks the Maxwell fitting of the ion beam. (c) and (d) are two wave modes calculated based on the ion distribution function, the frequency and growth rate are shown by black and blue solid lines and the black dash lines mark the ion Alfven speed $\left(V_{A}\right)$.

\section{Discussion and summary}

Taking account of the properties presented above, a possible candidate of the ion scale waves is the kinetic Alfven wave (KAW) [Hasegawa and Chen, 1976]. KAW 
can be generated by ion-ion streaming [Winske and Omidi, 1992; Yin et al., 2007], and carry a considerable amount of energy away from the fast plasma sheet flows [Chaston et al., 2012]. KAW occurs when the perpendicular scale of the Alfven wave [Alfven, 1942] approaches the ion cyclotron scale, and the frequency of KAW is of the order of $f_{c i}$. One important feature of the KAW is that it can generate parallel electric field, and we interpret the enhanced parallel electron phase space density is a result of trapping and acceleration of the electrons by the parallel electric field [Hui and Seyler, 1992; Kletzinlg, 1994; Artemyev et al., 2015]. However, the electric field data during the wave interval is complicated and could have included noise, making it difficult to establish the properties of the KAW with certainty. Therefore, we leave this issue for future studies.

In this event, the enhancement of the field-aligned component only appears in the parallel direction and no counter-streaming beams are observed. We interpret this character as an effect of the magnetic field configuration on the tailward side behind anti-dipolarization front (at 033000UT in Figure 1a), where the magnetic field is connected to the interplanetary magnetic field, and the field-aligned populations will not bounce back.

In the earth's magnetosphere, whistler mode waves can be generated by electron temperature anisotropy $\left(T_{\perp}>T_{\|}\right)$[Kennel and Petschek, 1966; Li et al., 2018], or electron beams [Sauer and Sydora, 2010; Ren et al., 2019]. In this study, the whistler mode waves are generated by electron populations streaming on one side along the magnetic field, and such a mechanism resembles the whistler heat flux instability (WHFI) [Tong et al., 2019a; Kuzichev et al., 2019] observed in the solar wind. The electron beta $\beta_{\mathrm{e}}$ (electron thermal pressure versus the magnetic pressure) in this event varied from 1 to 6 (Figure 1h), within the statistical range of the instability observed in the solar wind [Tong et al., 2019b], and the properties of these whistler 
waves that include the frequency range, right-hand circular polarization, and quasi-parallel propagation, are all similar to that generated by the solar wind WHFI [Tong et al., 2019a]. All these properties support the similarity of generation mechanism of the whistler mode waves discussed in this paper to the WHFI. As far as we know, the heat flux instability have not been reported in the earth's magnetosphere. Hence, the observations in this study give information on the heat flux instability exhibited in the distant magnetotail but with the help of ion scale waves.

Zhao et al., [2017] have shown that modulation of whistler mode waves also occurs near the neutral sheet region of distant magnetotail. However, the wave excitation and modulation mechanisms are different from the observations presented in this article. Near the neutral sheet region, the whistler mode waves are generated by temperature anisotropy of the electron flat-top distributions, and the ion scale waves could confine the generation and propagation of the whistler mode waves near the magnetic field valley. In this study, similar processes were also observed from 03:30:10 UT to 03:30:30 UT when the spacecraft crossed the neutral sheet region (Figure 1). Since the major conclusions are similar, the detail analysis was omitted here. These observations imply that the modulation of whistler mode waves by ion scale waves can take place with different mechanisms in the plasma sheet.

To reiterate, we have observed wave modulations between whistler mode waves and ion scale waves in the plasma sheet at $\sim 54 R_{\mathrm{E}}$. The ion scale waves, most likely kinetic Alfven waves, are excited by the interaction of the ion beam and the background ions. The ion scale waves periodically generated field-aligned electron populations, which drove whistler mode waves at each cycle of the ion scale waves. These observations have suggested that plasma dynamics on electron and ion scales can be coupled through waves. 


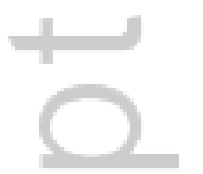

This article is protected by copyright. All rights reserved. 


\section{Acknowledgements}

This work was supported by the National Nature Science Foundation of China grant 41731068. We acknowledge NASA contract NAS5-02099 and V. Angelopoulos for use of data from the ARTEMIS mission. Specifically, we thank C. W. Carlson and J. P. McFadden for the ESA data, D. Larson and R. P. Lin for the SST data, A. Roux and O. LeContel for the SCM data; and K.-H. Glassmeier, U. Auster, and W. Baumjohann for the FGM data provided under the lead of the Technical University of Braunschweig and with financial support through the German Ministry for Economy and Technology and the German Center for Aviation and Space (DLR) under contract 50 OC 0302. All the data used in this paper are available online at http://themis.ssl.berkeley.edu/index.shtml. 


\section{References:}

Alfven, H., (1942), Existence of electromagnetic-hydromagneticwaves, Nature, 150, 405-406.

Angelopoulos, V., et al., (1992), Bursty bulk flows in the inner central plasma sheet, J. Geophys. Res., 97, 4027-4039.

Angelopoulos, V. (2008), The THEMIS Mission, SPACE SCI REV, 141(1-4), 5-34.

Angelopoulos, V. (2011), The ARTEMIS Mission, SPACE SCI REV, 165, 3-25.

Artemyev, A. V., R. Rankin, and M. Blanco (2015), Electron trapping and acceleration by kinetic Alfven waves in the inner magnetosphere, J. Geophys. Res. Space Physics, 120, 10,305-10,316, doi:10.1002/2015JA021781.

Artemyev, A. V., Angelopoulos, V., Runov, A., \& Vasko, I. Y. (2017). Hot ion flows in the distant magnetotail: ARTEMIS observations from lunar orbit to -200 RE. Journal of Geophysical Research: Space Physics, 122, 9898-9909. https://doi.org/10.1002/2017JA024433

Auster, H. U., et al. (2008), The THEMIS fluxgate magnetometer, Space Sci. Rev., 141(1-4), 235-264.

Baumjohann, W., G. Paschmann, H. Luhr, (1990), Characteristics of High-Speed Ion Flows in the Plasma Sheet, J. Geophys. Res., 95, A4, 3801-3809.

Chaston, C. C., J. W. Bonnell, L. Clausen, and V. Angelopoulos (2012), Energy transport by kinetic-scale electromagnetic waves in fast plasma sheet flows, J. Geophys. Res., 117, A09202, doi:10.1029/2012JA017863.

Colpitts, C. A., C. A. Cattell, M. Engebretson, M. Broughton, S. Tian, J. Wygant, A. Breneman, and S. Thaller (2016), Van Allen Probes observations of cross-scale coupling between electromagnetic ion cyclotron waves and higher-frequency wave modes, Geophys. Res. Lett., 43, 11,510-11,518, doi:10.1002/2016GL071566.

Cully, C.M., R.E. Ergun, K. Stevens, A. Nammari, J. Westfall, (2008), The THEMIS Digital Fields Board, Space Sci Rev, 141: 343-355,

DOI:10.1007/s11214-008-9417-1.

Donovan, E., et al. (2008), Simultaneous THEMIS in situ and auroral observations of a small substorm, Geophys. Res. Lett., 35, L17S18, doi:10.1029/2008GL033794. 
Bame, S. J., J. R. Asbridge, H. E. Felthauser, E. W. Hones, and I. B. Strong (1967), Characteristics of the plasma sheet in the Earth's magnetotail, J. Geophys. Res., 72(1), 113-129, doi:10.1029/JZ072i001p00113.

Drake, J. F., O. V. Agapitov, and F. S. Mozer (2015), The development of a bursty precipitation front with intense localized parallel electric fields driven by whistler waves, Geophys. Res. Lett., 42, doi:10.1002/2015GL063528.

Fu, H. S., et al., (2011), Fermi and betatron acceleration of suprathermal electrons behind dipolarization fronts, Geophys. Res. Lett., 38, L16104, doi:10.1029/2011GL048528.

Gabrielse, C., V. Angelopoulos, C. Harris, A. Artemyev, L. Kepko, and A. Runov (2017), Extensive electron transport and energization via multiple, localized dipolarizing flux bundles, J. Geophys. Res. Space Physics, 122, 5059-5076, doi:10.1002/2017JA023981.

Hasegawa, A. and L. Chen, (1976), Kinetic processes in plasma heating by resonant mode conversion of Alfvén wave, The Physics of Fluids, 19, 1924, doi: 10.1063/1.861427.

Hui, C.-H., and C. E., Seyler, (1992), Electron Acceleration by Alfven Waves in the Magnetosphere, JOURNAL OF GEOPHYSICAL RESEARCH, 97(A4), 3953-3963.

Karpman, V. I., and R. N. Kaufman, (1982), Whistler wave propagation in density ducts, J. Plasma Physics, 27(2), 225-238.

Keika, K., M. Spasojevic, W. Li, J. Bortnik, Y. Miyoshi, and V. Angelopoulos (2012), PENGUIn/AGO and THEMIS conjugate observations of whistler mode chorus waves in the dayside uniform zone under steady solar wind and quiet geomagnetic conditions, J. Geophys. Res., 117(A07212), doi:10.1029/2012JA017708.

Kennel, C. F., \& Petschek, H. E. (1966). Limit on stable trapped particle fluxes. Journal of Geophysical Research, 71(1), 1-28.

Khotyaintsev, Yu.V., et al., (2011), Plasma Jet Braking: Energy Dissipation and Nonadiabatic Electrons, PHYSICAL REVIEW LETTERS, 106, 165001.

Kiehas, S. A., Runov, A., Angelopolos, V., Hietala, H., and Korovinksiy, D. (2018), Magnetotail fast flow occurrence rate and dawn-dusk asymmetry at $\mathrm{X}_{\mathrm{GSM}} \sim-60 \mathrm{R}_{\mathrm{E}}$, Journal of Geophysical Research: Space Physics, 123. https://doi.org/10.1002/2017JA024776

Kletzinlg, C. A., (1994), Electron acceleration by kinetic Alfven waves, J. Geophys. Res., 99(A6), 11,095-11,103. 
Kuzichev, I. V., Vasko, I. Y. , Soto-Chavez, A. R. , Tong, Y. , Artemyev, A. V. , \& Bale, S. D. , et al. (2019). Nonlinear evolution of the whistler heat flux instability. The Astrophysical Journal. Doi:10.3847/1538-4357/ab3290.

Li, W., R. M. Thorne, J. Bortnik, Y. Nishimura, and V. Angelopoulos (2011), Modulation of whistler mode chorus waves: 1 . Role of compressional Pc4-5 pulsations, J. Geophys. Res., 116, A06205, doi:10.1029/2010JA016312.

Li, W., Shen, X. - C., Ma, Q., Capannolo, L., Shi, R., Redmon, R. J., et al. (2019). Quantification of energetic Electron precipitation driven by plume whistler mode waves, Plasmaspheric hiss, and exohiss. Geophysical Research Letters, 46, 36153624. https://doi.org/10.1029/2019GL082095

Li, L. Y., J. Yu, J. B. Cao, D. Zhang, X. H. Wei, Z. J. Rong, J. Y. Yang, and H. S. Fu (2013), Rapid loss of the plasma sheet energetic electrons associated with the growth of whistler mode waves inside the bursty bulk flows, J. Geophys. Res. Space Physics, 118, 7200-7210, doi:10.1002/2013JA019109.

Li, S.-S., J. Liu, V. Angelopoulos, A. Runov, X.-Z. Zhou, and S. A. Kiehas (2014), Antidipolarization fronts observed by ARTEMIS, J. Geophys. Res. Space Physics, 119, 7181-7198, doi:10.1002/2014JA020062.

Li, J., Bortnik, J., An, X., Li, W., Russell, C. T., Zhou, M., et al. (2018). Local excitation of whistler mode waves and associated Langmuir waves at dayside reconnection regions. Geophysical Research Letters, 45, 8793-8802. https://doi.org/10.1029/2018GL078287.

McFadden, J. P., C. W. Carlson, D. Larson, M. Ludlam, R. Abiad, B. Elliott, P. Turin, M. Marckwordt, and V. Angelopoulos (2008), The THEMIS ESA plasma instrument and in-flight calibration, Space Sci. Rev., 141(1-4), 277-302.

Mozer, F. S., Agapitov, O. V., Blake, J. B., \& Vasko, I. Y. ( 2018). Simultaneous observations of lower band chorus emissions at the equator and microburst precipitating electrons in the ionosphere. Geophysical Research Letters, 45, 511- 516. https://doi.org/10.1002/2017GL076120

Nagai, T., et al., (1998), Structure and dynamics of magnetic reconnection for substorm onsets with Geotail observations, JOURNAL OF GEOPHYSICAL RESEARCH, 103 (A3), 4419-4440.

Nakamura, R., et al. (2002), Motion of the dipolarization front during a flow burst event observed by Cluster, GEOPHYS RES LETT, 29 (194220).

Ren, Y., L. Dai, W. Li, X. Tao, C. Wang, B. Tang, et al. (2019). Whistler waves driven by field-aligned streaming electrons in the near-Earth magnetotail 
reconnection. Geophysical Research Letters, 46, 5045-054.

https://doi.org/10.1029/2019GL083283.

Ronnmark, K. (1982), WHAMP - waves in homogeneous, anisotropic,

multicomponent plasmas, KGI Rep. 179, Kiruna Geophys. Inst., Kiruna, Sweden.

Roux, A., O. Le Contel, C. Coillot, A. Bouabdellah, B. de la Porte, D. Alison, S.

Ruocco, and M. C. Vassal (2008), The search coil magnetometer for THEMIS, Space

Sci. Rev., 141(1-4), 265-275.

Runov, A., et al., (2009), THEMIS observations of an earthward-propagating dipolarization front, GEOPHYS RES LETT, 36 (L14106).

Runov, A., V. A. Sergeev, V. Angelopoulos, K.-H. Glassmeier, and H. J. Singer (2014), Diamagnetic oscillations ahead of stopped dipolarization fronts, J. Geophys. Res. Space Physics, 119, 1643 - 1657, doi:10.1002/2013JA019384.

Sauer, K., and R. D. Sydora (2010), Beam-excited whistler waves at oblique propagation with relation to STEREO radiation belt observations, Ann. Geophys., 28, 1317-1325, doi:10.5194/angeo-28-1317-2010.

Sazhin, S. S., and M. Hayakawa (1992), Magnetospheric chorus emissions: A review, Planet. Space Sci., 40(5), 681-697, doi:10.1016/0032-0633 (92)90009-D.

Slavin, J. A., R. P. Lepping, J. Gjerloev, D. H. Fairfield, M. Hesse, C. J. Owen, M. B. Moldwin, T. Nagai, A. Ieda, and T. Mukai (2003), Geotail observations of magnetic flux ropes in the plasma sheet, J. Geophys. Res., 108(A1), 1015, doi:10.1029/2002JA009557.

Sergeev, V. A., I. A. Chernyaev, S. V. Dubyagin, Y. Miyashita, V. Angelopoulos, P. D. Boakes, R. Nakamura, and M. G. Henderson (2012), Energetic particle injections to geostationary orbit: Relationship to flow bursts and magnetospheric state, J. Geophys. Res., 117, A10207, doi:10.1029/2012JA017773.

Streltsov, A. V., M. Lampe, W. Manheimer, G. Ganguli, and G. Joyce (2006), Whistler propagation in inhomogeneous plasma, J. Geophys. Res., 111, A03216, doi:10.1029/2005JA011357.

Summers, D., Y. Omura, S. Nakamura, and C. A. Kletzing (2014), Fine structure of plasmaspheric hiss, J. Geophys. Res. Space Physics, 119, 9134-9149, doi:10.1002/2014JA020437.

Tenerani, A., O. Le Contel, F. Califano, F. Pegoraro, P. Robert, N. Cornilleau-Wehrlin, and J. A. Sauvaud, (2012), Coupling Between WhistlerWaves and Ion-Scale SolitaryWaves: Cluster Measurements in the Magnetotail During a 
Substorm, PHYSICAL REVIEW LETTERS, 109, 155005, DOI:

10.1103/PhysRevLett.109.155005.

Thorne, R. M., Binbin Ni, Xin Tao, Richard B. Horne \& Nigel P. Meredith (2010), Scattering by chorus waves as the dominant cause of diffuse auroral precipitation, Nature, 467, 943-946, doi:10.1038/nature09467.

Tong, Y. G., I. Y. Vasko, P. Marc, F. S. Mozer, S. D. Bale, A. V. Artemyev, V. Krasnoselskikh (2019a), Whistler Wave Generation by Halo Electrons in the Solar Wind. The Astrophysical Journal Letters. 870. Doi:10.3847/2041-8213/aaf734.

Tong, Y. , Vasko, I. Y., Artemyev, A. V. , Bale, S. D. , \& Mozer, F. S. . (2019b). Statistical study of whistler waves in the solar wind at $1 \mathrm{AU}$, doi:10.3847/1538-4357/ab1f05.

Tyler, E., Breneman, A., Cattell, C., Wygant, J., Thaller, S., \& Malaspina, D. (2019). Statistical occurrence and distribution of high-amplitude whistler mode waves in the outer radiation belt. Geophysical Research Letters, 46, 2328-2336. https://doi.org/10.1029/2019GL082292.

Viberg, H., Y. V. Khotyaintsev, A. Vaivads, M. André, H. S. Fu, and N. Cornilleau-Wehrlin (2014), Whistler mode waves at magnetotail depolarization fronts, J. Geophys. Res. Space Physics, 119, 2605-2611, doi:10.1002/2014JA019892.

Xie, H., \& Yong, X. (2016), PDRK: A General Kinetic Dispersion Relation Solver for Magnetized Plasma, Plasma Science and Technology, 18, 2, 97. doi: 10.1088/10090630/18/2/01

Yin, L., D. Winske, W. Daughton, and K. J. Bowers, (2007), Kinetic Alfvén waves and electron physics. I. Generation from ion-ion streaming, Phys. Plasmas 14, 062105, DOI: 10.1063/1.2734950.

Wilson, L. B., III, C. A. Cattell, P. J. Kellogg, J. R. Wygant, K. Goetz, A. Breneman, and K. Kersten (2011), The properties of large amplitude whistler mode waves in the magnetosphere: Propagation and relationship with geomagnetic activity, Geophys. Res. Lett., 38, L17107, doi:10.1029/2011GL048671.

Winske, D., and N. Omidi, (1992), Electromagnetic Ion/Ion Cyclotron Instability: Theory and Simulations, J. Geophys. Res., 97(A10), 14779-14799.

Zhang, X., Angelopoulos, V., Artemyev, A. V., \& Liu, J. (2018), Whistler and electron firehose instability control of electron distributions in and around dipolarizing flux bundles. Geophysical Research Letters, 45, 9380-9389. https://doi.org/10.1029/2018GL079613. 
Zhang. Xu, V. Angelopoulos, A. V. Artemyev, Jiang Liu (2019a), Energy transport by whistler waves around dipolarizing flux bundles, Geophysical Research Letters, doi: 10.1029/2019GL084226.

Zhang, X.-J., Chen, L., Artemyev, A. V., Angelopoulos, V., \& Liu, X. (2019b). Periodic excitation of chorus and ECH waves modulated by ultralow frequency compressions. Journal of Geophysical Research: Space Physics, 124. https://doi.org/10.1029/2019JA027201.

Zhao, D., et al. (2016), Electromagnetic disturbances observed near the dip region ahead of dipolarization front, Geophys. Res. Lett., 43, 3026-3034, doi:10.1002/2016GL068033.

Zhao, Duo, et al., (2017), Electron flat-top distributions and cross-scale wave modulations observed in the current sheet of geomagnetic tail, Physics of Plasmas, 24, 082903 (2017); doi: 10.1063/1.4997765.

Zhou, X. Z., et al. (2010), Accelerated ions ahead of earthward propagating dipolarization fronts, JOURNAL OF GEOPHYSICAL RESEARCH-SPACE PHYSICS, 115(A00I03).

Zhou, X.-Z., D.-X. Pan, V. Angelopoulos, J. Liu, A. Runov, S.-S. Li, J.-Z. Li, Q.-G. Zong, S.-Y. Fu (2015), Ion acceleration and reflection on magnetotail antidipolarization fronts, Geophys. Res. Lett., 42, doi:10.1002/2015GL065865. 


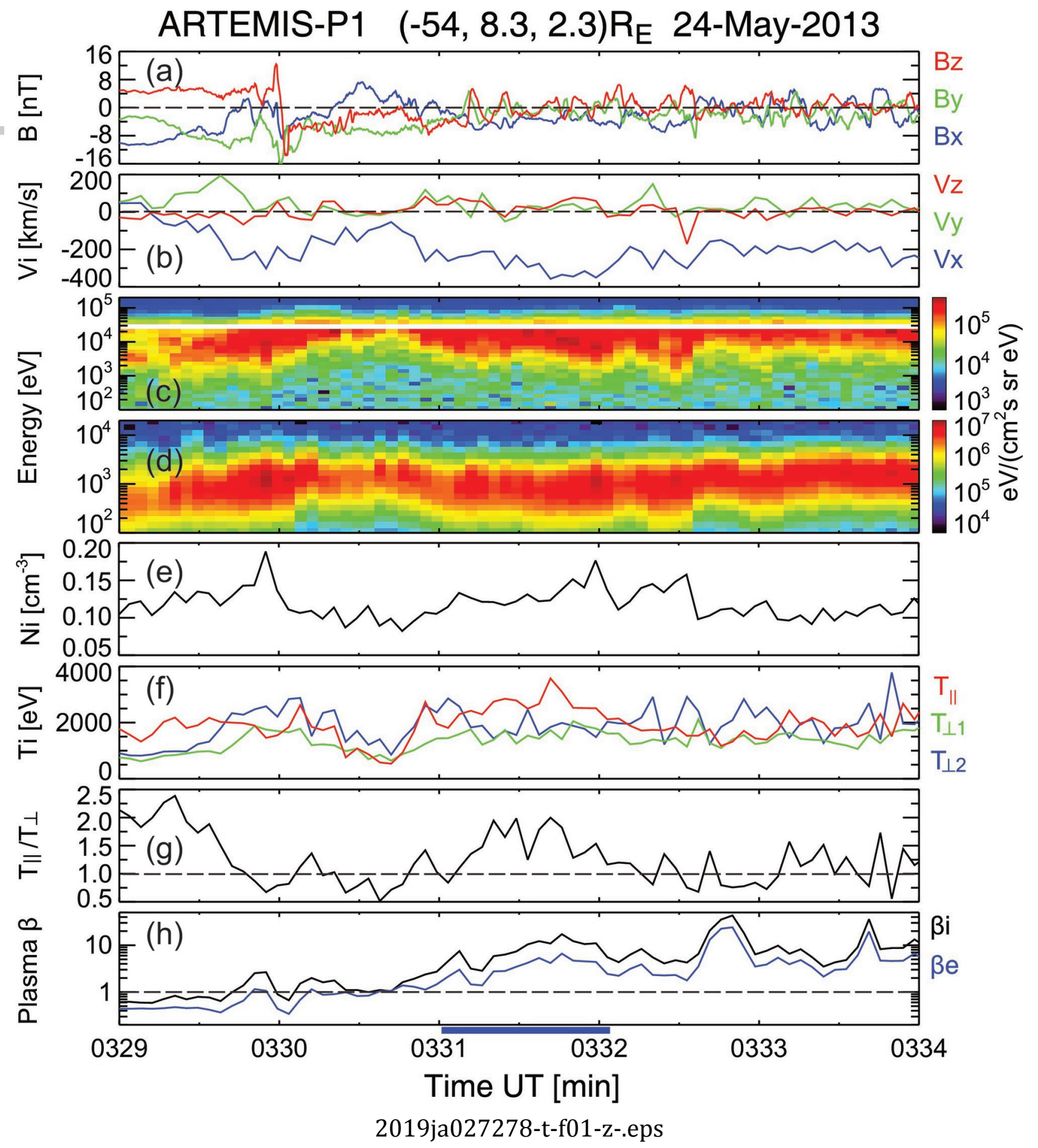

This article is protected by copyright. All rights reserved. 

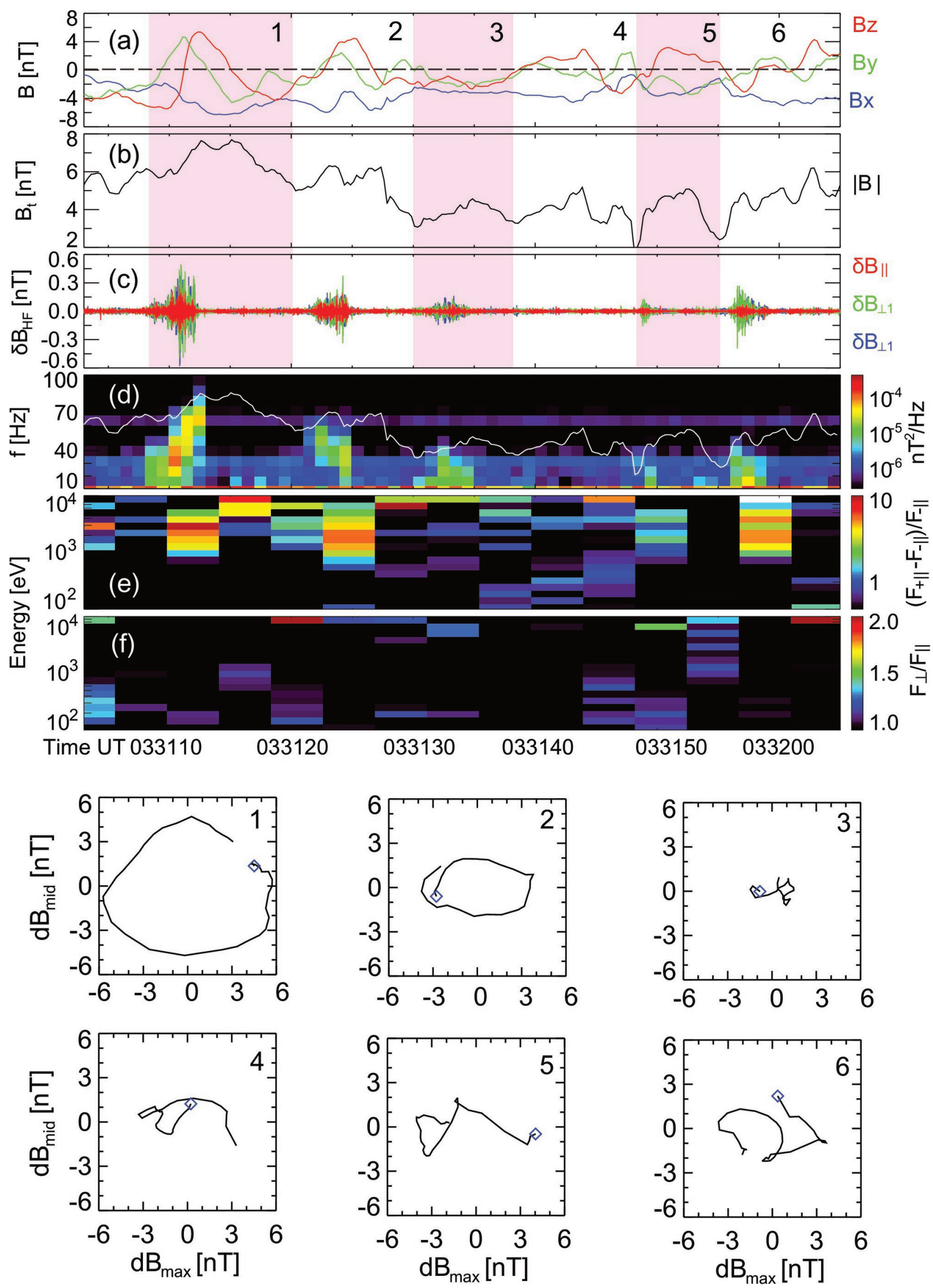

2019ja027278-t-f02-z-.eps 

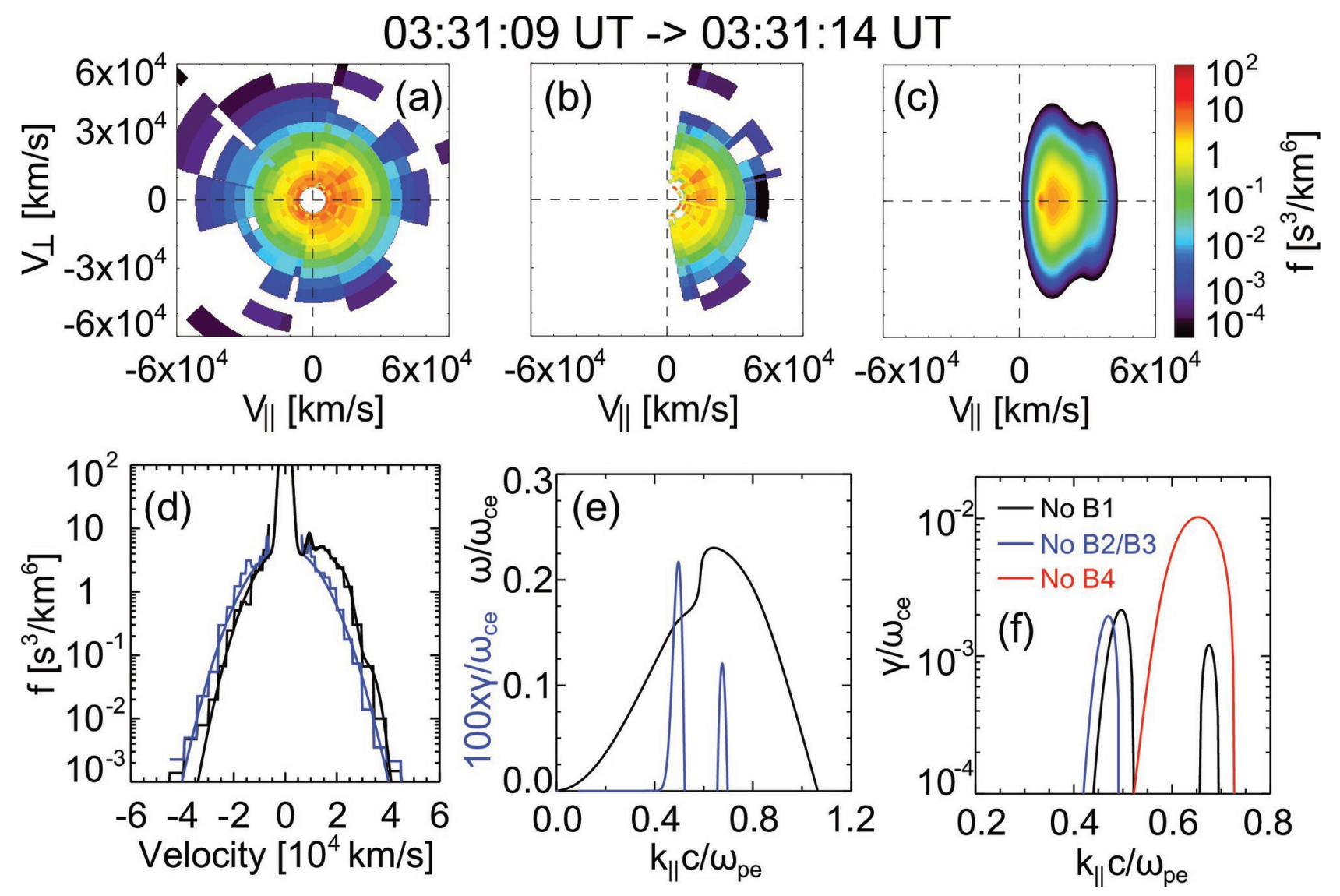

2019ja027278-t-f03-z-.eps 

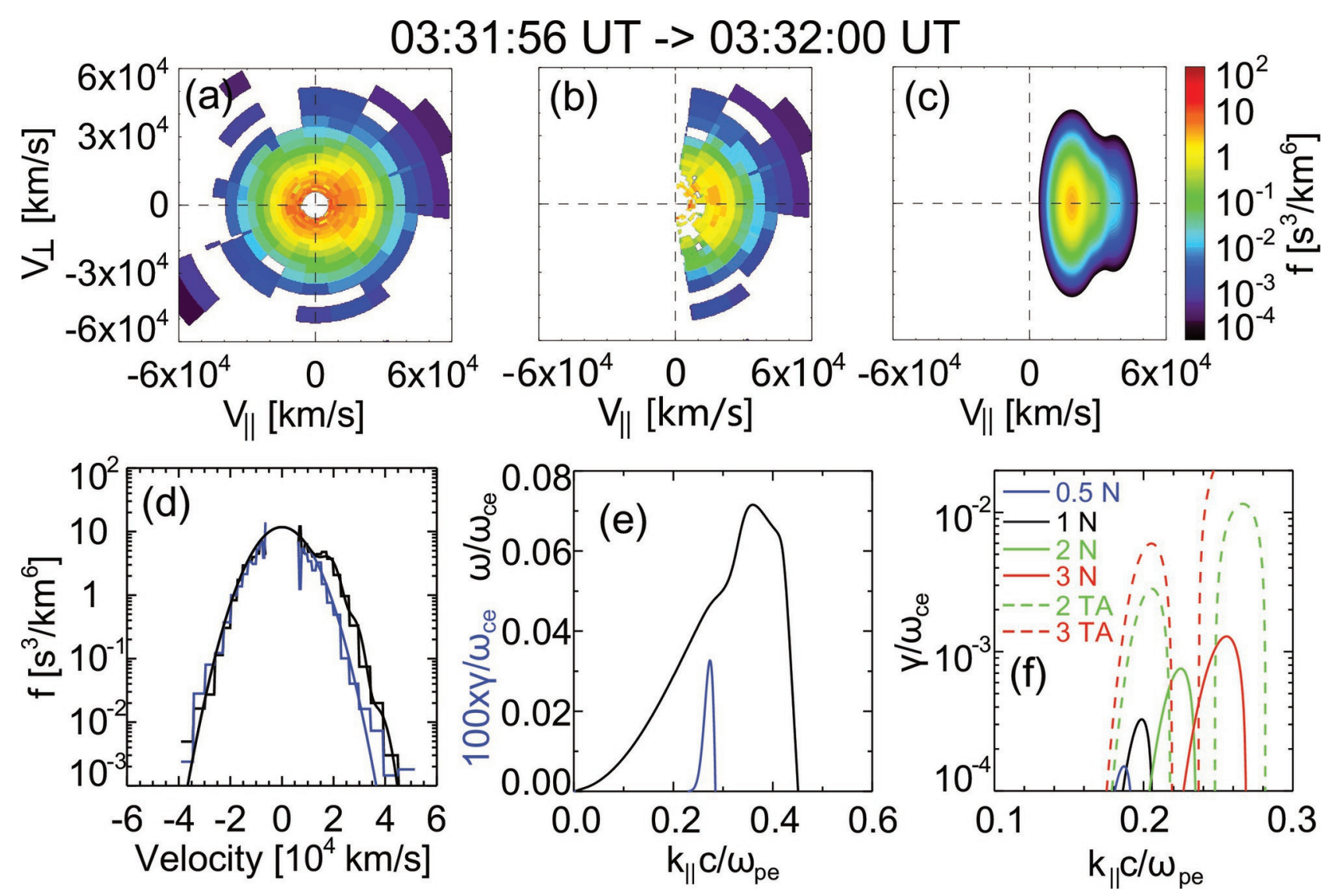

2019ja027278-t-f04-z-.eps 

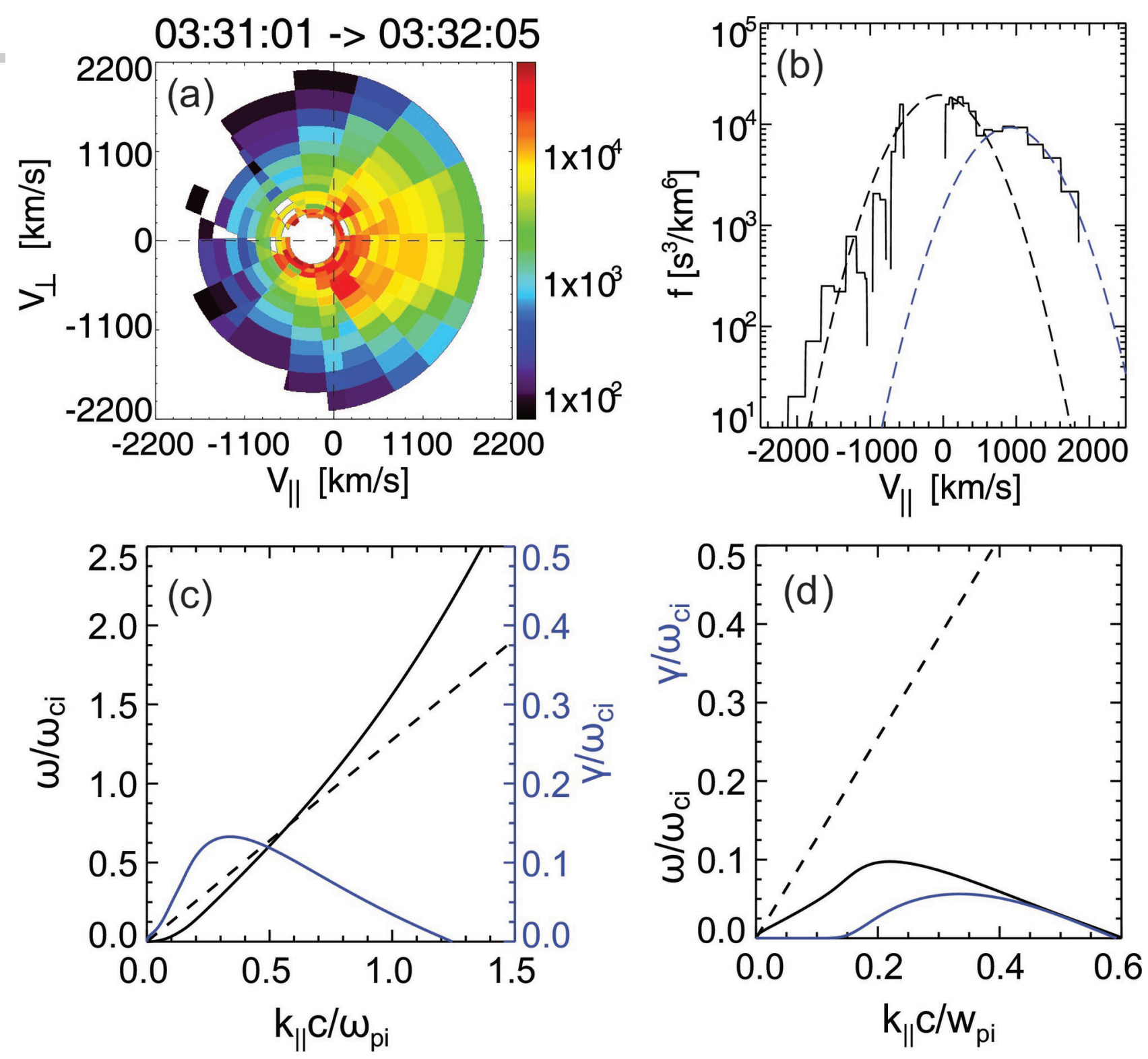

2019ja027278-t-f05-z-.eps 


\begin{tabular}{|c|c|c|c|c|}
\hline \multicolumn{5}{|c|}{033109 UT -033114 UT, $|\boldsymbol{B}|=6.5 \mathrm{nT}$, Whistler mode wave } \\
\hline Components & $\mathrm{N}\left(\mathrm{cm}^{-3}\right)$ & $\overline{V_{0}(\mathrm{~km} / \mathrm{s})}$ & $\mathrm{T}_{\|}(\mathrm{eV})$ & $\mathrm{T}_{\perp} / \mathrm{T}_{\|}$ \\
\hline Hot electrons & 0.0435 & -4130.4 & 384 & 1.4 \\
\hline Cold electron & 0.0258 & -4130.4 & 10 & 1 \\
\hline Electron beam 1 & 0.0004 & 5204.6 & 5 & 6 \\
\hline Electron beam 2 & 0.0155 & 10258.6 & 50 & 10 \\
\hline Electron beam 3 & 0.0068 & 17166.6 & 60 & 6 \\
\hline Electron beam 4 & 0.0003 & 28045.6 & 55 & 10 \\
\hline Ion & 0.0923 & 0 & 2000 & 1 \\
\hline \multicolumn{5}{|c|}{033156 UT -- 033200 UT, $|\boldsymbol{B}|=4.5$ nT, Whistler mode wave } \\
\hline Hot electrons & 0.11 & -2011.3 & 404 & 1 \\
\hline Electrons beam 1 & 0.012 & 16532.7 & 59 & 8 \\
\hline Electrons beam 2 & 0.00075 & 25817.7 & 41 & $\overline{8}$ \\
\hline Electrons beam 3 & 0.0001 & 34862.7 & 64 & 8 \\
\hline Ions & 0.12285 & 0 & 2000 & 1 \\
\hline \multicolumn{5}{|c|}{ 03:31:00 UT -- 03:32:05 UT, $|\boldsymbol{B}|=4.9$ nT, Ion scale wave } \\
\hline Hot Ion & 0.047 & -189 & 2219 & 1.56 \\
\hline Cold Ion & 0.059 & -189 & 100 & 1 \\
\hline Ion Beam & 0.026 & 780 & 2346 & 1.66 \\
\hline Electrons & 0.132 & $\overline{0}$ & 487 & 1 \\
\hline
\end{tabular}

This article is protected by copyright. All rights reserved. 